\title{
Reducing the risk of Chlamydia trachomatis transmission: male circ umcision or a female vaccine?
}

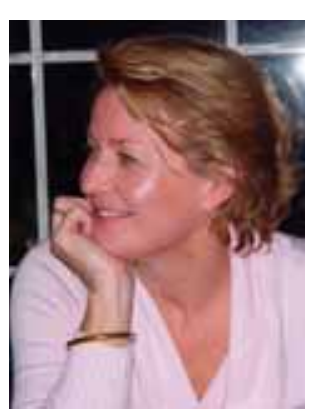

LaiseM Hafiner

School of Life Sciences \& Institute of $\mathrm{H}$ ealth \& Biomedical Innovation (IH BI), Q ueensiand U niversity of Technology, 2 George Street, GPO Box 2434, Brisbane, QLD 4001, Australia

Tel.: +61 731381305 ; Fax: +61 731381 534; I.hafner@qut.edu.au future, part of fso

\section{'...vaccine research is headed rapid ly towa rds ha messing the Trojan horse that is Chlamydia trachomatis'.}

$M$ ale circumcision - is it the answer to reducing the risk of acquiring and transmitting venereal diseases such as Chlamydia [1]? As a woman, the aforementioned strategy might appear to be one of the easiest solutions to deal with one of the oldest public health problems in the world. A relatively poor understanding of the regulation of the immune response of the female genital tract, the lack of adjuvants capable of targeting vaccines to the genital mucosa, our limited knowledge of which chlamydial antigens induce protective responses, the lack of a system to genetically manipulate chlamydiae and the observation that immune responses may be involved in pathogenesis, all suggest that the development of an efficacious chlamydial vaccine is a nigh-on-impossible task. As a female scientist, however, who has been working in the field of Chlamydia for the past 18 years, it is my opinion that vaccine research is headed rapidly towards harnessing the Trojan horse that is Chlamydia trachomatis.

Chlamydia trachomatis serovars $\mathrm{D}-\mathrm{K}$ are important causes of sexually transmitted disease (STD) both in males and in females in developed and developing countries Most recent international reports (as of early 2007) indicate that Chlamydia accounts for an estimated 92 million of the 500 million annual STDs worldwide affecting both women (50 million) and men (42 million) [2]. There is currently no vaccine available for either gender.

The human pathogen $C$. trachomatis has been described as a microbial Trojan horse as it is a highly evolved obligate intracellular bacterium that has developed unique mechanisms to evade the host immune system during infection. The surface of the infectious chlamydial elementary body $(E B)$ is covered with antigenically diverse surface proteins that help avoid detection by antibodies [3]. The intracellular replication of chlamydiae, within a membrane-bound inclusion, limits exposure of the organism to the immune system [4]. Once inside this intracellular niche, chlamydiae are thought to interact with the host via a type three secretion apparatus, which allows the chlamydiae to alter host-cell functions.

C. trachomatis serovars D-K are associated with acute urethral syndrome, urethritis and cervicitis in women and epididymitis, acute proctitis acute proctocolitis in men. However, up to $75 \%$ of infections are often asymptomatic and subclinical in both males and females [5-8]. An estimated $40 \%$ of untreated genital chlamydial infections lead to sequelae, including pelvic inflammatory disease that can result in infertility, ectopic pregnancy and chronic pelvic pain in women [9]. Chlamydia infections in males can cause urethritis, prostatitis and epididymitis that can lead to infertility [10].

'The ideal vaccine would be one that protects against multiple serovars and prevents chronic infection sequelae...'

$M$ any infections are asymptomatic and hence effective treatments with antibiotics such as doxycyline and azithromycin cannot be commenced. These were until recently defined as effective therapy - but if high chlamydial loads are found in vivo then it is possible that women treated with these antibiotics are at increased risk of treatment failure [11]. Thus, the development of an efficacious vaccine would appear to be the most fiscally prudent approach to controlling chlamydial genital infections. Indeed, it has been shown by modeling studies that even a partially protective prophylactic vaccine that is capable of preventing severe disease sequelae would be a suitable shortterm goal to control chlamydial infections [12]. The ideal vaccine would be one that protects against multiple serovars and prevents chronic infection sequelae, including blindness, pelvic inflammatory disease and infertility.

Antichlamydial protective immunity is essentially mediated by a T-helper type 1 (Th1), response that is dependent upon the presentation of antigen via major histocompatibility 
complex (M HC) class II molecules. While antibody-secreting cells are not critical components of the primary effector response, they have been shown to be important for clearance of reinfection. Thus, an ideal vaccine would be one capable of inducing both a strong Th1 T-cell response and a strong mucosal antibody response. Currently, there are very few efficacious vaccine candidates that have been identified and characterized. M ore specifically, there is only a limited number of known $\mathrm{T}$-cell antigens processed and presented by the human leukocyte antigen (H LA) class II molecules. This type of antigen is going to be essential to the development of an efficacious chlamydial vaccine.

Because it is currently not feasible to genetically modify chlamydiae in order to produce safe attenuated strains, the recombinant subunit approach is the one considered most likely to be successful. Among the chlamydial antigen candidates showing the greatest promise is a $40 \mathrm{kD} \mathrm{a}$ outer membrane antigen, known as major outer membrane protein MOMP, as well as the polymorphic outer membrane proteins ( $P O M P$ ), the PorB family of membrane antigens, an AD P/AT P translocase, a PGP3 plasmid protein and others [13-15].

The total number of characterized antigens is somewhat limited, possibly due to most research being focused on the development of M O M P as a single antigen vaccine. $O$ ne of the main foci in chlamydial vaccine design has been on the use of the MOMP as a single antigen component. MOMP constitutes approximately $60 \%$ of the total protein mass of the bacterial outer membrane [16]. Interestingly, the last few years have seen an increase in the number of novel chlamydial antigens being reported, possibly reflecting a change in attitude towards chlamydial vaccine development and the fact that there are at least ten entire chlamydial genomes now available from The Institute for G enomic Research (TIGR).

\section{'...predic ting immunogenic ity based on a protein'sfunction is now becoming redundant'.}

There is a great deal of diversity in the type of protein that has been reported as being immunogenic during chlamydial infection. There are a number of classical vaccine targets, such as the polymorphic outer membrane proteins and type three secretion system proteins (LCrD, LcrE, CopN , YscT and Ysc]) [17-20]. However, there are also a number of unusual vaccine targets that have been shown to be immunogenic and even able to confer protection, such as enolase, a ribosomal protein, a histone-like protein, RN A polymerase and chlamydial protease-like activity factor (CPAF) [21-23]. The discovery of immunogenic targets, such as $\mathrm{N} \mathrm{rdB}$, enolase, ribosomal proteins and RN A polymerase, suggest predicting immunogenicity based on a protein's function is now becoming redundant.

Using expression library immunization we have screened the genome of Chlamydia muridarum and identified several promising gene fragments that protected genetically immunized mice by eliciting immune responses that contributed to increasing the clearance of live chlamydial infection from the genital tracts of these animals. The proteins we have identified as partially protective include a putative outer membrane protein (TC0512), two conserved hypothetical proteins (TC 0757 and TC 0767) an adherence factor protein (TC0439), a U vrD /REP helicase family protein (TC0490) and an ATP-dependent CIp protease, ATP-binding subunit C IpC protein (TC 0559).

The fact that we have identified genes/proteins presumably not exposed on the surface of C hlamydia that are protective is not unexpected and concordant with findings of earlier studies reporting protection against $M$ ycobacterium avium subsp. paratuberculosis infection provided by transport and chaperone proteins using expression library immunization [24]. H ousekeeping genes have also been reported to mediate high levels of protection in the mouse pneumonia model of Chlamydial infection [25]. Resolution of primary Chlamydial infection is known to require MHC class IIrestricted CD 4+ Th1 and M HC class I-restricted $\mathrm{CD} 8^{+} \mathrm{T}$ cells mediated by the proinflammatory cytokine interferon (IFN)- $\gamma$ [26-29]. 0 ur results are therefore plausible since peptide fragments of the adherence factor protein or the REP helicase protein would have the opportunity to bind to the M HC class II complex and be recognized by the immune system in this complex.

In another of our recent studies [30], we have identified a number of unique vaccine candidates using a novel in silico approach. In an attempt to overcome H LA polymorphism, the whole chlamydial genome was screened for proteins containing epitopes predicted to bind multiple H LA class II molecules (i.e., predicted 'promiscuous' T-cell epitopes). A wide range of H LA class II molecules were used in this screen to identify vaccine antigens that could potentially offer broad and ethnically balanced 
population coverage. This analysis identified a number of novel targets and was validated by the identification of a known chlamydial $T$-cell epitope.

Clinical persistence is a key concept in the pathogenesis of chlamydial infections. Sterility caused by genital infection with $C$. trachomatis and coronary artery disease associated with Chlamydia pneumoniae are both caused by inflammation-based pathology. Chlamydial antigens may persist for prolonged periods and there is evidence that these noncultivable forms may lead to continued inflammation.

During its intracellular developmental cycle, C. trachomatis maintains this intracellular niche since it possesses the tryptophan synthase virulence gene that has coevolved with its human host. This virulence factor acts as a primary mechanism for the invading pathogen to hide from the host's primary line of defense [31]. Chlamydiae also express a type III secretion system (T 3SS) that secretes antihost (effector) proteins into the inclusion membrane known as inclusion membrane-localized proteins (Inc-proteins) and host cytosol that allow the organisms to establish and maintain the permissive intracellular niche in the host $[32,33]$. Persistence of the microorganisms by hiding from the immune system thus raises the question of optimal therapy as well as presenting a unique challenge to the development of effective vaccines.

O ur own group has several potential chlamydial persistence antigens that can be tested in a vaccine that targets both the acute and the persistent stages of infection - both of which are the result of infection of the host cell cytoplasmic membrane and abnormal growth and persistence of the organism - two of the known stages of the chlamydial developmental cycle [34]. It would appear that a multisubunit vaccine would be

most effective, especially considering recent evidence reporting that a novel, multiple subunit vaccine induced a higher frequency of Th1 cells against $C$. trachomatis compared with a single subunit construct [35]. Thus, it would appear that an effective chlamydial vaccine will need to have multiple subunits, some of which are directed against acute antigens and others directed against persistent antigens.

'...c andidate vaccine targets that induce partial immunity may well be able to induce sterilizing immunity...'

It will of course be imperative for any novel vaccine trials to evaluate what effect(s) immunizing against persistence antigens will have in situations where chlamydial infections of animals/people are either inapparent (subclinical) or persistently progressing (chronic).

There are now several novel vaccine candidates that can elicit partial protection against genital infection in various animal models of genital chlamydial infection. Novel antigens could be used either individually or as part of a multisubunit construct with the appropriate delivery methods aimed to enhance uptake, expression and presentation of antigens. U sed with improved effective delivery systems and potent adjuvants, candidate vaccine targets that induce partial immunity may well be able to induce sterilizing immunity if a higher frequency of Thl cells can be induced after primary immunization with the novel chlamydial antigens.

So to answer my initial question, no, male circumcision is not the definitive answer. Vaccine research is indeed headed rapidly towards harnessing the Trojan horse of $\mathrm{C}$. trachomatis. In fact, a female vaccine will only be one half of the story.

Bibliography

1. Castellsague X, Peeling RW, Franceschi S et al.: IARC M ulticenter Cervical $C$ ancer Study Group. Chlamydia trachomatis infection in female partners of circumcised and uncircumcised adult men. Am. J. Epidemiol. 162(9), 907-916 (2005).

2. WH O: G lobal prevalence and incidence of selected curable sexually transmitted infections. O verview and Estimates. WH O Geneva, Switzerland (2001).

3. Brunham RC, Plummer FA, Stephens RS: Bacterial antigenic variation, host immune response, and pathogen-host coevolution. Infect. Immun. 61, 2273-2276 (1993).
4. Fields KA, H ackstadt $\mathrm{T}$ : The chlamydial inclusion: escape from the endocytic pathway. Annu. Rev. Cell D ev. Biol. 18, 221-245 (2002).

5. Schacter f: NAATs to diagnose Chlamydia trachomatis genital infection: a promise still unfulfilled. Exp.Rev. M ol. Diag. 1(2), 137-144 (2001).

6. Johnston RE, Green TA, Schacter J et al.: Evaluation of nucleic acid amplification tests as reference tests for Chlamydia trachomatis infections in asymptomatic men. J. Clin M icro. 38(12), 4382-4386 (2000).

7. Stamm WE: Chlamydia trachomatis infections of the adult. In: Sexually Transmitted D iseases H olmes KK, Sparling PF, M ardh P-A (Eds). M cGraw-H ill, N ew York, USA (1999).
8. Chen M Y, D onovan B: Screening for genital Chlamydia trachomatis infection: are men the forgotten reservoir? M ed. J. Aust. 179(3), 124-125 (2003).

9. Westrom I, Joesoef $R$, Reynolds $G$, $H$ adgu $A$, Thomson SE: Pelvic inflammatory disease and infertility: a cohort study of 1844 women with laparoscopically verified disease and 657 control women with normal laparoscopy results. Sex. Transm. Dis. 19, 185- 192 (1992).

10. Wagenlehner FM, Weidner W, N aber KG: Chlamydial infections in urology. World J. U rol. 24(1), 4-12 (2006). 
11. H orner $P: T$ The case for further treatment studies of uncomplicated genital Chlamydia trachomatis infection. Sex Trans. Inf. 82, 340-343 (2006).

12. de la $M$ aza $M A$, de la $M$ aza $L M$ : A new computer model for estimating the impact of vaccination protocols and its application to the study of Chlamydia trachomatis genital infections. Vaccine 13(1), 119-127 (1995).

13. D onati M, Sambri V, Comanducci M et al.: D N A immunization with pgp3 gene of Chlamydia trachomatis inhibits the spread of chlamydial infection from the lower to the upper genital tract in C $3 \mathrm{H} / \mathrm{H}$ eN mice. Vaccine 21, 1089-1093 (2003).

14. Kawa DE, Schachter J, Stephens RS: Immune response to the Chlamydia trachomatis outer membrane protein PorB. Vaccine 22, 4282-4286 (2004).

15. M urdin AD, D unn P, Sodoyer R: U se of a mouse lung challenge model to identify antigens protective against Chlamydia pneumoniae lung infection. J. Infect. D is. 181(Suppl. 3), S544-S551 (2000).

16. Brunham RC, Peeling RW: Chlamydia trachomatis antigens: role in immunity and pathogenesis. Infect. Agents D is. 3, 218-233 (1994).

17. Sharma J, Zhong Y, D ong F, Piper JM, Wang G, Zhong G: Profiling of human antibody responses to $C$ hlamydia trachomatis urogenital tract infection using microplates arrayed with 156 chlamydial fusion proteins. Infect. Immun. 74, 1490-1499 (2006).

18. Thorpea C, Edwardsa L, Snelgrovea R et al.: D iscovery of a vaccine antigen that protects mice from Chlamydia pneumoniae infection Vaccine 25(12), 2252-2260 (2007).

19. Tammiruusu A, Penttila T, Lahesmaa R, Sarvas $M$, Puolakkainen $M$, Vuola JM : Intranasal administration of chlamydial outer protein $\mathrm{N}$ (CopN) induces protection against pulmonary Chlamydia pneumoniae infection in a mouse model. Vaccine 25(2), 283-290 (2007).
20. Capo S, Nuti S, Scarselli M : Chlamydia pneumoniae genome sequence analysis and identification of H LA-A2-restricted CD $8^{+}$ $T$ cell epitopes recognized by infectionprimed T cells. Vaccine 23, 5028-5037 (2005).

21. Goodall JC, Beacock-Sharp H, D eane KH, Gaston JS: Recognition of the 60 kilodalton cysteine-rich outer membrane protein $\mathrm{OMP} 2$ by CD $4^{+} \mathrm{T}$ cells from humans infected with Chlamydia trachomatis. Clin. Exp. Immunol. 126, 488-493 (2001).

22. M urthy $A K, C$ ong $Y, M$ urphey $C$ et al.: Chlamydial protease-like activity factor induces protective immunity against genital chlamydial infection in transgenic mice that express the human H LA-D R 4 allele. Infect. Immun. 74(12), 6722-6729 (2006).

23. M urthy AK, Chambers JP, M eier PA, Zhong $G$, Arulanandam BP: Intranasal vaccination with a secreted chlamydial protein enhances resolution of genital Chlamydia muridarum infection, protects against oviduct pathology, and is highly dependent upon endogenous gamma interferon production. Infect. Immun.. 75(2), 666-676 (2007).

24. H untley JF, Stabel JR, Paustian M L, Reinhardt TA, Bannantine JP: Expression library immunization confers protection against $M$ ycobacterium avium subsp. paratuberculosis infection. Infect. Immun. 73(10), 6877-6884 (2005).

25. Stemke-H ale K, Kaltenboeck B, $D$ eG raves FJ et al.: Screening the whole genome of a pathogen in vivo for individual protective antigens. Vaccine 23(23), 3016-3025 (2005).

26. M orrison RP, Feilzer K, Tumas D B: Gene knockout mice establish a primary protective role for major histocompatibility complex class II-restricted responses in Chlamydia trachomatis genital tract infection. Infect. Immun. 63(12), 4661-4668 (1995).

27. M orrison SG, M orrison RP: In situ analysis of the evolution of the primary immune response in murine Chlamydia trachomatis genital tract infection. Infect. Immun. 68(5), 2870-2879 (2000).
28. H awkins RA, Rank RG, Kelly KA: Chlamydia trachomatis-specific Th2 clone does not provide protection against a genital infection and displays reduced trafficking to the infected genital mucosa. Infect. Immun. 70(9), 5132-5139 (2002).

29. Igietseme JU, Ramsey KH, M agee D M , Williams D M , Kincy TJ, Rank RG : Resolution of murine chlamydial genital infection by the adoptive transfer of a biovar-specific, Th1 lymphocyte clone. Reg. Immunol. 5(6), 317-324 (1993).

30. Barker C: Identification and characterisation of novel candidates for a vaccine against chlamydial genital tract infection. PhD Thesis, Institute of $\mathrm{H}$ ealth and Biomedical Innovation (IH BI), Q ueensland University of Technology (QUT), Brisbane, Q ueensland, Australia (2007).

31. M CC larty G, C aldwell H D, N elson DE: Chlamydial interferon- $\gamma$ immune eevasion influences infection tropism. Curr. 0 pin. Microbiol. 10(1), 47-51 (2007).

32. H ackstadt T, Scidmore-Carlson M A, Shaw EI, Fischer ER: The Chlamydia trachomatis IncA protein is required for homotypic vesicle fusion. Cell. M icrobiol. 1(2), 119-130 (1999).

33. Fields KA, M ead DJ, D ooley CA, $\mathrm{H}$ ackstadt $\mathrm{T}$ : Chlamydia trachomatistype III secretion: evidence for a functional apparatus during early-cycle development. M ol. M icrobiol. 48(3), 671-683 (2003).

34. Abdel Rahman YM, Belland RJ: The chlamydial developmental cycle. FEM S M icro. Rev. 29, 949-959 (2005).

35. Eko FO, HeQ, Brown T et al.: A novel recombinant multisubunit vaccine against Chlamydia. J. Immunol. 173, 3375-3382 (2004).

Affiliation

- Louise M H afner, Associate Professor School of Life Sciences \& Institute of $H$ ealth \& Biomedical Innovation (IH BI), Q ueensland U niversity of Technology, 2 G eorge Street, GPO Box 2434, Brisbane, Q LD 4001, Australia Tel.: +61 731381 305; Fax: +61 731381 534; I.hafner@qut.edu.au 